\title{
Strengthening Telepsychiatry's Role in Clinical Care and Education
}

\author{
Richard Balon • Eugene V. Beresin • John H. Coverdale • \\ Alan K. Louie • Laura Weiss Roberts
}

Received: 10 November 2014 / Accepted: 13 November 2014/Published online: 5 December 2014

(C) Academic Psychiatry 2014

Telepsychiatry provides psychiatric care and support for individuals separated by distance from providers, through the engagement of electronic and other communication and information technology [1-3]. Also referred to as "telemental health" [3], telepsychiatry has been used for decades and continues to evolve. Actually, in 1973, Thomas Dwyer [4] described the use of "interactive television"-possible at that time because of the introduction of "cable TV" systems - as an "enormous leap forward in carrying psychiatric help to the population." Telepsychiatry has indeed become increasingly popular in the USA and other countries, such as Australia, Canada, Finland, Israel, New Zealand, and the UK [1, 3]. The first comprehensive curriculum for teaching telepsychiatry to psychiatry residents was proposed on the pages of this journal 15 years ago [5].

Telepsychiatry has been promoted as a more practical and less expensive way to diagnose and manage mental and substance use disorders in remote communities that may not have a local psychiatrist [6]. Telepsychiatry has been used in the identification and treatment of diverse disorders, for example, psychosocial support and medication management in the care of patients with schizophrenia, group therapy sessions for veterans with posttraumatic stress disorder, and evaluation and consultation neuropsychology services [1]. Provision of care across distance is a patient-centered clinical strategy, we

R. Balon $(\bowtie)$

Wayne State University, Detroit, MI, USA

e-mail: rbalon@wayne.edu

E. V. Beresin

Harvard Medical School, Boston, MA, USA

J. H. Coverdale

Baylor College of Medicine, Houston, TX, USA

A. K. Louie • L. W. Roberts

Stanford University School of Medicine, Stanford, CA, USA suggest, in which it can bring treatment into a patient's own living space. This approach reduces the burden and costs experienced by those who must travel, for example, to urban centers, in order to receive appropriate specialty and subspecialty care. For these reasons, telepsychiatry has the important potential to make psychiatric services accessible to those individuals and special populations who might otherwise miss out, including many patients who are economically disadvantaged or otherwise marginalized. Indeed, current discussions in integrated behavioral health suggest that telepsychiatry makes more accessible new communityand evidence-based approaches for treating major mental disorders [7] and co-occurring disorders, often through integration with primary care services at Federally Qualified Health Centers, for example. Consultation and follow-up delivered by telepsychiatry was found to produce outcomes equivalent to services provided face-to-face in one Canadian study [6], with telepsychiatry services being at least $10 \%$ less expensive per patient than services provided in person.

Beyond direct clinical care, telepsychiatry facilitates innovative clinical training. Through engagement of the telepsychiatry platform, it is possible to offer an ancillary educational function for clinicians managing complex cases who may not have the in-house faculty to support ongoing professional development and quality improvement. The combined effect of clinical training and clinical care in psychiatry represents an exciting next evolutionary step in telepsychiatry. Efforts to deliver and amplify muchneeded expertise across populations and to strengthen the skills and knowledge of current and future independent practitioners are needed, given the prevalence of mental conditions and the insufficiency of current systems of care. Telepsychiatry can thus enable mental health of individuals and populations directly and indirectly through clinical care and clinical training approaches. 
In 2014, the American Medical Association approved recommendations for telemedicine. We believe that the psychiatry profession will soon create similar recommendations for telepsychiatry. So far, the American Telemedicine Association has established guidelines for videoconferencing-based telemental health [8], and Shore [9] has written a thoughtful and practical overview of administrative and clinical issues involving telepsychiatry. Such guidelines are especially and increasingly important in the USA, where roughly $25 \%$ of the population lives in the rural and frontier contexts that represent $75 \%$ of the nation's land mass. Under the Affordable Care Act, US systems of care will have to adapt to decreased reimbursement levels and increased population management imperatives. As the system evolves, the locus of treatment may occur more often in medical homes in the community and less often at tertiary care, resource-rich teaching hospitals. As Dwyer [4] wrote more than 40 years ago, optimal medical practice in the USA requires that the wisdom of community-based providers be shared with academic centers and that these same practitioners have easier access to the new knowledge generated in these centers. This bidirectional exchange of wisdom, new knowledge, and experience, he believed, was the foundation of early telepsychiatry. We believe that this exchange is the essence of its value for telepsychiatry-based innovative education in the future as well.

\section{In This Issue}

For telepsychiatry to assume a more prominent role in the delivery of care for people living with mental disorders and co-occurring conditions in the future, we will have to better educate and prepare our residents for the best possible use of this technology. Four articles in this issue of Academic Psychiatry [10-13] extend our understanding of the use of telepsychiatry in clinical practice and psychiatric education. As we can see from the papers in this issue, there is still a long way to go before telepsychiatry can become better established.

Hoffman and Kane [10] surveyed 183 programs regarding a curriculum in telepsychiatry. Fewer than $50 \%$ of responding programs in their study reported that their residents were involved in telepsychiatry either through a formal curriculum or informal exposure. Only about $26 \%$ of programs reported having formal curriculum in telepsychiatry. Although the response rate in this study was low $(25 \%)$, these numbers suggest that there is still a lot to do to add to residents' experiences in this area. Sunderji and colleagues [11] suggest in their narrative review that we need a more evidence-based approach to telepsychiatry training. The authors pointed out that residents' interest and intent to practice via telepsychiatry currently exceeds what is offered during training. Hoffman and Kane [10] also posit that the inclusion of telepsychiatry in residency education could instill values such as social accountability for care of marginalized populations and cultural sensitivity, shift attitudes toward technology, and expose trainees to interprofessional care and novel models of care.

DeGaetano et al. [12], in an educational case report, describe the development and experience of teaching telepsychiatry to third-year psychiatry residents on a 6month outpatient telepsychiatry rotation at the Palo Alto Veterans Administration Hospital. During this rotation, residents were scheduled for three to four patients during a 3-h clinic and using telepsychiatry during their half-day addiction clinic (they were also involved in other clinics during their outpatient rotation). Attending physicians sat in with the residents during the initial sessions, but later on, most attending physicians provided a mix of in-person supervision with secure instant messaging and telephone supervision. In a follow-up survey, most participating residents and attending physicians felt very positive about the experience; residents wanted it to continue as a required rotation.

As telepsychiatry has been establishing its place in providing clinical services, the use of telepsychiatry in psychiatric education, for example, in training to deliver psychotherapy services [14], is another important trend of particular interest to our readers. A special section of Psychotherapy (Chicago, IL, USA) was devoted to video and Internet applications in psychotherapy supervision and training $[14,15]$. This growing trend aims to rectify deficiencies in training in resource-poor educational programs.

In keeping with this theme, Katzman and colleagues [13] described the use of teletechnology in using resources outside their department for training residents in psychodynamic psychotherapy. Four psychoanalysts from around the nation who were known to their faculty organized 90 -min case conferences for the residents in the University of New Mexico program. Contact with teletechnologies was described as "just like being there," and it "led more to a sense of delight than it has to frustration." When learning the paradigm of intensive short-term dynamic psychotherapy, a group of faculty and residents connected to a trainer at a distant site (Dalhousie University, Halifax, Nova Scotia). A faculty member also videotaped confidential, password-protected psychotherapy sessions for which patients gave consent and which utilized particular software and technological devices for distant weekly supervision. This innovative activity enabled the psychiatrist to develop expertise in this method of psychotherapy for sharing with others in the local community. Such methods and technologies could also presumably benefit by bringing teachers to programs in need of more teaching in the clinical neurosciences [16] or in other areas such as psychiatric ethics or evidence-based psychiatry. 


\section{Looking Ahead}

Hilty and colleagues state, "The future use of telepsychiatry and other technologies is uncertain. For telepsychiatry, its curve of growth or decline will depend on how well programmes are organised, adapt and deal with potential pitfalls" [1, p. 544]. Adapting telepsychiatry in widely varying clinical settings requires overcoming a number of practical obstacles. As a practicing telepsychiatrist, Daniel Knoedler reminds us [17], "Telepsychiatry is a team sport," in that it requires on-site staff support, technical support and expertise, and a costly initial setup. He points out that telepsychiatry is better for the psychiatrist who works from home, from a warm place in the winter, but its value must be considered in relation to the needs of individual patients. He also points out the implications that telepsychiatry has for the doctor-patient relationship, including privacy issues, especially in cases when patients are seen in their home environment or when telepsychiatry sessions are offered in settings or through technological supports that are insufficiently secured. Ongoing studies of the outcomes, costs, and benefits of the provision of telepsychiatry involving patients, their families, and particular communities will be essential if telepsychiatry is to become a useful tool in the transformation of our health system.

Although the resource expenditures associated with telepsychiatry in clinical and educational settings may be largely "up front"- the costs to set up proper programs and software, the resources to purchase equipment, and the investment of time and intellectual effort to create the educational modules - much could be done to reduce costs in the future. Teaching activities such as lectures, consultations, and case discussions could be recorded and coupled with online didactic curricula, and then "packages" of real-time (e.g., video consultations or supervisions) and recorded materials could be provided effectively to various residency programs. Outcome studies of the benefits of using telepsychiatry as a teaching and learning tool for both residents and faculty will need to be conducted, so that we as psychiatric educators and clinicians appreciate the value of this technology in the professional development of our workforce.

Additionally, legal issues will need to be addressed for the use of telepsychiatry in the real-time conduct of consultation, diagnostic evaluation, and perhaps most importantly, ongoing treatment of patients across state lines. There is ongoing debate about where the locus of treatment exists when a clinician is caring for patients across state lines. Questions to consider include the following: Is this treatment in the home state of the patient, in which the treating physician is not licensed, or in the home state of the clinician? Would reciprocity in state licenses be needed for such practices? Further, will we need increased Health Insurance Portability and Accountability Act-protected media to allow for such treatment? In which electronic medical record would the patient's chart reside, the outside clinician's or that of the home institution where the patient is seen? Finally, would the clinician be able to bill insurance companies for the provision of remote care? At present, controversy arises because remote telepsychiatric treatment may not be considered an "office visit" and costs are typically borne by the patient, as is typical in private practice. Would institutionally obtained insurance policies cover both the clinician and patient receiving training and care? Could remote clinicians and consultants be reimbursed for their clinical practice?

Although many of these questions remain unanswered, their resolution is critical if telepsychiatry is to become an integral part of our system of care. These legal issues may not be applied to the use of telepsychiatry for educational purposes with the informed consent of any patients who are volunteers, but they certainly will have to be addressed for ongoing clinical care.

Telepsychiatry and other technologies have an extraordinary potential for improving psychiatric care and training. Video-teleconferencing, use of telepsychiatry for didactic teaching and case conferences, psychotherapy and psychopharmacology telesupervision, psychotherapy teletraining, teleconsultations, and other teleteaching (e.g., continuing medical education) will combine various resources and expertise and allow us to substantially extend the reach of teaching, to improve its methods and quality, and, we hope, to demonstrate improved patient outcomes.

Acknowledgments The authors thank Gabrielle Termuehlen and Madeline Lane-McKinley for their editorial assistance in preparing this paper.

Disclosures On behalf of all the authors, the corresponding author states that there are no conflicts of interest.

\section{References}

1. Hilty DM, Luo JS, Morache C, Marcelo DA, Nesbitt TS. Telepsychiatry: an overview for psychiatrists. CNS Drugs. 2002;16: $527-48$.

2. Deslich S, Stec B, Tomblin S, Coustasse A. Telepsychiatry in the 21st century: transforming healthcare with technology. Perspect Health Inf Manag. 2013;10:1f.

3. Hilty DM, Ferrer DC, Parish MB, Johnston B, Callahan EJ, Yellowlees PM. The effectiveness of telemental health: a 2013 review. Telemed J E Health. 2013;19:444-54.

4. Dwyer TF. Telepsychiatry: psychiatric consultation by interactive television. Am J Psychiatry. 1973;130:865-9.

5. Oesterheld JR, Travers HP, Kofoed L, Hacking DM. An introductory curriculum on telepsychiatry for psychiatric residents. Acad Psychiatry. 1999;23:165-7.

6. O’Reilly R, Bishop J, Maddox K, Hutchinson L, Fisman M, Takhar J. Is telepsychiatry equivalent to face-to-face psychiatry? Results from a randomized controlled equivalence trial. Psychiatr Serv. 2007;58: $836-47$. 
7. Falloon IRH, Fadden G. Integrated mental health care: a comprehensive community approach. Cambridge: University Press; 1993.

8. Yellowlees P, Shore J, Roberts L. American Telemedicine Association: practice guidelines for videoconferencing-based telemental healthOctober 2009. Telemed J E Health. 2010;16(10):1074-89.

9. Shore JH. Telepsychiatry: videoconferencing in the delivery of psychiatric care. Am J Psychiatry. 2013;170:256-62.

10. Hoffman P, Kane JM. Telepsychiatry education and curriculum development in residency training. Acad Psychiatry. 2014. doi:10.1007/ s40596-013-0006-6.

11. Sunderji N, Crawford A, Jovanovic M. Telepsychiatry in graduate medical education: a narrative review. Acad Psychiatry. 2014. doi:10. 1007/s40596-014-0176-x

12. DeGaetano N, Greene CJ, Dearaujo N, Lindley SE. A pilot program in telepsychiatry for residents: initial outcomes and program development. Acad Psychiatry. 2014. doi:10.1007/s40596-014-0122-y.
13. Katzman J, Abbass A, Coughlin P, Arora S. Building connections through teletechnologies to augment resident training in psychodynamic psychotherapy. Acad Psychiatry. 2014. doi:10.1007/s40596014-0108-9.

14. Manring J, Greenberg JP, Gregory R, Gallinger L. Learning psychotherapy in the digital age. Psychotherapy (Chic). 2011;48:119-26.

15. Wolf AW. Internet and video technology in psychotherapy supervision and training. Psychotherapy (Chic). 2011;48:179-81.

16. Coverdale J, Balon R, Beresin EV, Louie AK, Tait GR, Goldsmith M, et al. Teaching clinical neuroscience to psychiatry residents: model curricula. Acad Psychiatry. 2014;38:111-5.

17. Knoedler DW. Telepsychiatry is a team sport. Psychiatr Times. 2014;XXXI(6):40A. Available at http://www.psychiatrictimes. com/telepsychiatry/telepsychiatry-team-sport. Accessed 10 Nov 2014. 